\title{
INCIDENCE AND RISK FACTORS FOR HEPATITIS IN PATIENTS RECEIVING ANTITUBERCULAR TREATMENT
}

\author{
Manish Kumar Singh, Mamatha. S, Rashee Jain, Amit Kumar Jha, Sanjay Kumar Nigam
}

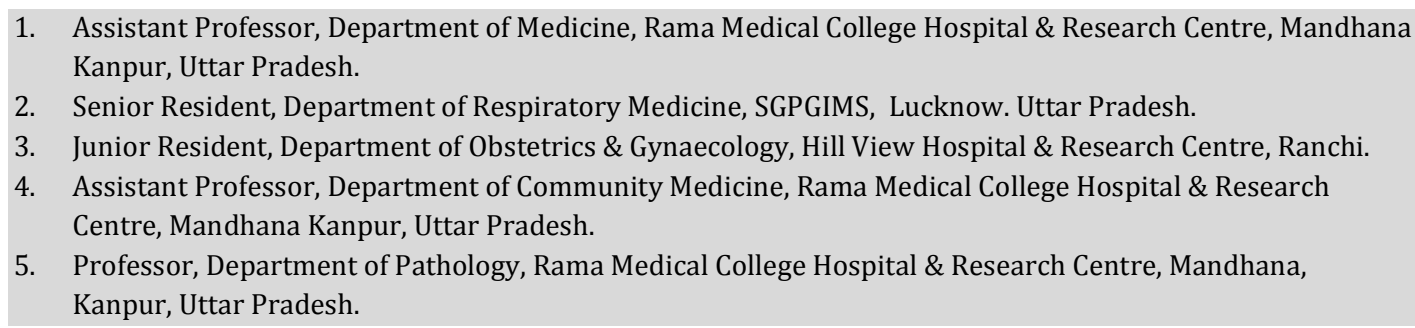

\section{CORRESPONDING AUTHOR}

Dr. M. K. Singh, House No. 206, Staff Quarter-2, Rama Medical College, Mandhana, Kanpur, Uttar Pradesh, E-mail: dr.manishkumarsingh@yahoo.com Ph: 00917376070025.

ABSTRACT: INTRODUCTION: Tuberculosis still remains a serious clinical problem. Standard first line drugs such as isoniazid, rifampicin, pyrazinamide, ethambutol or streptomycin. Of these, isoniazid, rifampicin and pyrazinamide have been observed to have hepatotoxic potential and drug induced hepatotoxicity (DIH) is an important and commonly encountered adverse effect with anti TB treatment 2, 3 . A higher risk of hepatotoxicity has been reported in Indian patients ${ }^{4-7}$ than in their western counterparts ${ }^{7-9}$.The reasons for the higher rate of hepatotoxicity in Indian patients are unclear. AIMS AND OBJECTIVES: 1 . To study the incidence of hepatitis in patients receiving antitubercular treatment as per Revised National Tuberculosis Control Programme.

2. To know the possible risk factors for the development of drug induced hepatotoxicity.

MATERIAL AND METHODS: In the prospective study, 100 freshly diagnosed pulmonary tuberculosis patients attending the medicine outpatient department or admitted in Rajendra Institute of Medical Sciences, Ranchi and attending RNTCP DOTS center in the hospital were taken up for study. RESULTS: Twenty eight patients (28\%) developed antituberculosis treatment induced hepatotoxicity which included $50 \%$ of cases in the age group of 51-60 years and $80 \%$ of cases in the age group of $>60$ years .Among patients who developed DIH $78.6 \%$ patients had pretreatments albumin $<3.5 \%$ gm/dl. $46.4 \%$ patients had history of high alcohol intake, 50\%patients had $\mathrm{BMI}<18,53.6 \%$ patients had radio logically severe disease.

CONCLUSION: The incidence of asymptomatic elevation of liver enzymes secondary to antituberculosis treatment is $23 \%$ and incidence of overt DIH is $5 \%$. Advanced age, high alcohol intake, BMI < 18, radiologically severe disease and pretreatment hypoalbuminemia are predisposing factors for the development of ATT induced hepatotoxicity.

KEYWORDS: Drug induced hepatitis, anti tubercular treatment, RNTCP

INTRODUCTION: Tuberculosis remains one of the world's serious clinical problems. Nearly one third of global population, i.e. two billion people, is infected with M. tuberculosis and at risk of developing the disease. More than $90 \%$ of global TB cases and deaths occur in the developing world; more than $75 \%$ of cases are in the most economically productive age group (15-54 yrs). 
Co-infection with the HIV significantly increases the risk of developing tuberculosis. At the same time, multidrug resistance, which is caused by poorly managed TB treatment, is growing problem of serious concern in many countries around the world ${ }^{1}$.The essential services needed to control tuberculosis, based on diagnosis and treatment of infectious cases and incorporating the essential management tools, were developed and packaged as the DOTS strategy in the early 1990s. In 1993, W.H.O. took the unique step of declaring tuberculosis to be world emergency. With this in mind came the Revised National Tuberculosis Control Programme (RNTCP), which was pilot tested in 1993.The RNTCP is an application of the W.H.O recommended strategy of Directly Observed Therapy Short course (DOTS) in India. On March 24, 1997 the Director General of WHO declared "the DOTS strategy for tuberculosis control represents the most important public health breakthrough of the decade, in terms of lives which will be saved". The number of countries adopting DOTS has increased dramatically over the decade, from a handful in 1990 to 148 in 2002. India now has the second largest DOTS Programme in the world. Presently standard first line drugs such as isoniazid, rifampicin, pyrazinamide, ethambutol or streptomycin are essential components of DOTS strategy. Of these, isoniazid, rifampicin and pyrazinamide have been observed to have hepatotoxic potential and drug induced hepatotoxicity (DIH) is an important and commonly encountered adverse effect with anti TB treatment ${ }^{3}, 4$. Several risk factors for the development of hepatotoxicity during short course therapy have been suggested. A higher risk of hepatotoxicity has been reported in Indian patients5-8 than in their western counterparts ${ }^{8-10}$.The reasons for the higher rate of hepatotoxicity in Indian patients are unclear.

\section{AIMS AND OBJECTIVES:}

1. To study the incidence of hepatic damage and overt hepatitis in patients receiving antitubercular treatment as per Revised National Tuberculosis control Programme (RNTCP).

2. To know the possible risk factors for the development of drug induced hepatotoxicity

MATERIAL \& METHODS: In the prospective study, 100 freshly diagnosed pulmonary tuberculosis patients males as well as females attending the medicine outpatient department or admitted in Rajendra Institute of Medical Sciences, Ranchi and attending RNTCP DOTS center in the hospital were taken up for study.

\section{INCLUSION CRITERIA:}

Patients diagnosed to have pulmonary tuberculosis with sputum smear positive for the first time

\section{EXCLUSION CRITERIA:}

1. Patients with extrapulmonary tuberculosis.

2. Patients of pulmonary tuberculosis who are defaulters, treatment failure cases and multidrug resistance cases.

3. Patients with abnormal baseline liver function tests.

4. Patients with cirrhosis of liver, acute viral hepatitis and/or gastrointestinal, renal or cardiac diseases.

STUDY AREA: Medicine outpatient department or admitted in Rajendra Institute of Medical Sciences, Ranchi, Jharkhand and attending RNTCP DOTS center in the hospital. 
METHODOLOGY: 100 patients with pulmonary tuberculosis diagnosed for the first time. Detailed history was taken and detailed clinical examination was done, including measurement of height, weight and BMI. Detailed history of high alcohol intake was noted.

RESULTS AND DISCUSSION: In our study, we enrolled 100 patients who are diagnosed to have pulmonary tuberculosis for the first time. These patients were given DOTS therapy (cat I, cat III) as per RNTCP guidelines. All patients had normal liver function tests before the initiation of therapy. Liver function tests were monitored at $2^{\text {nd }}, 4^{\text {th }}$ and $8^{\text {th }}$ week of the treatment to note the elevation in serum bilirubin and liver transaminase levels which indicate the antituberculosis treatment induced hepatotoxicity. The results hereby are discussed under separate headings for each variable.

These results indicate that advancing age is an independent risk factor for drug induced hepatotoxicity consistent with previous reports. The patients in the age group of $>50$ years are 5.36 times more likely to have liver damage as compared to the patients in the age group of $<50$ years $(\mathrm{p}=0.007)$.

HIGH ALCOHOL INTAKE : So the patients with history of high alcohol intake are 4.46 times more likely to have elevated liver enzymes as compared to non-alcoholics while receiving ATT $(\mathrm{p}=0.005),(\mathrm{OR}=4.81)$. Among non-alcoholics, sex is not a risk factor for elevation of liver enzymes.

BODY MASS INDEX: The body mass index can be independent factor to predict the risk of ATT induced liver damage. The patients with BMI $<18$ are 2.42 times more likely to have elevated liver enzymes.

SEVERITY OF DISEASE: The patients who present with radio logically severe disease are 4.38 times more likely to have drug induced liver damage than patients presenting with milder disease $(p=0.001,0 R=4.38)$. In DIH group $(n=5) 4(80 \%)$ cases had severe disease on their chest x-ray.

HYPOALBUMINEMIA: The patients presenting with pre-treatment hypoalbuminemia are 14 times more likely to have ATT induced liver damage than patients presenting with normal serum albumin levels.

In this study 28 (28\%) cases showed the evidence of antituberculosis treatment (ATT) induced liver damage in the form of elevation of serum bilirubin and transaminase levels above normal. Among these 5 (5\%) cases developed overt drug induced hepatotoxicity (DIH) as defined above and remaining 23 (23\%) cases had asymptomatic elevation of serum liver enzymes and bilirubin levels. The remaining $72(72 \%)$ cases didn't show any significant change in their serum bilirubin and/or transaminase levels as compared to pre-treatment levels.

DISCUSSION: Isoniazid, rifampicin and pyrazinamide have been observed to have hepatotoxic potential and drug induced hepatotoxicity (DIH) is an important and commonly encountered adverse effect with anti TB treatment 2, 3 Several types of drug induced liver damage have been described. Mechanisms of Drug Induced Hepatotoxicity include-Idiosyncratic damage, Dose dependent toxicity, Induction of hepatic enzymes, Drug induced acute hepatitis, Allergic reactions. 
Specific Patterns of Hepatic Damage include- Disruption of intracellular calcium homeostasis, Cholestatic damage, Interruption of transport pumps and loss of villous processes, Reactions involving cytochrome p-450 system, Activation of apoptotic pathways and programmed cell death and Inhibition of mitochondrial function. So early recognition of risk factors with close follow up of patients receiving ATT and subjecting them to repeated liver function tests will significantly reduce morbidity and mortality and improve the compliance of the patients receiving ATT.

CONCLUSION: The incidence of drug induced hepatotoxicity (DIH) in patients receiving DOTS therapy (cat I \& III) is 5\%. Peak incidence of ATT induced liver damage occurs at third and fourth week of therapy. Patients in the age group of $>50$ years are 5.36 times more likely to have drug induced liver damage than patients in the age group of $<50$ years. Male alcoholics are 4.46 times more likely to have drug induced liver damage as compared to male non-alcoholics. Patients with BMI $<18$ are 2.4 times more likely to develop ATT induced liver damage than patients with BMI $>18$. Patients with pretreatment hypoalbuminemia are 14 times more likely to have ATT induced liver damage \& Patients presenting with radiologically severe disease are 4.38 times more likely to develop ATT induced liver damage. So, in our study we found that advancing age, low BMI, history of high alcohol intake, radio logically severe disease and pretreatment hypoalbuminemia are independent risk factors for development of anti-tubercular treatment induced hepatotoxicity.

\section{BIBLIOGRAPHY:}

1. Espinal MA et al. Global trends in resistance to anti tuberculosis drugs, NEJM, 2001, 344(17): 1294-1303.

2. Girling DJ. The hepatic toxicity of antitubercular regimens containing isoniazid, rifampicin and pyrazinamide. Tubercle 1978:59: 13-32.

3. Gangadharam PR. Isoniazid, rifampicin and hepatotoxicity (editorial). Am Rev Respir dis 1986; 133; 963-65.

4. Parthasarathy R, Sarma GR, Janardhanam B, Ramachandran P, Santha T. Hepatic toxicity in south Indian patients during treatment of tuberculosis with short course regimens containing isoniazid,rifampicin and pyrazinamide. Tubercle 1986:67:99-108.

5. Purohit SD, Gupta PR, Sharma TN, Gupta DN, Chawla MP. Rifampicin and hepatotoxicity. Indian J Tuberc 1983:30:107-109.

6. Taneja DP, Kaur D. Study on hepatotoxicity and other side effects of antituberculosis drugs. J Indian Med Assoc 1990:88:278-280

7. Mehta S. Malnutrition and drugs; clinical implications. DevPharmacolTher1990 ;15:159-165.

8. Snider DE, Long MW, Cross FS, Farer LS. Six months isoniazid and rifampicin therapy for pulmonary tuberculosis: report of a United States Public Health Service cooperative trial. Am Rev Respir Dis 1984:129:573-579.

9. DuttAK, Moers D, Stead WW. Short course chemotherapy for tuberculosis with mainly twice weekly isoniazid and rifampicin:Community physician's seven year experience with mainly out patients. Am J Med 1984;77;233-242.

10. British Thoracic and Tuberculosis Association; Short course chemotherapy in pulmonary tuberculosis. Lancet 1975; I;119-124. 
TABLE - 1 ASSOCIATION OF AGE WITH DRUG INDUCED LIVER DAMAGE

\begin{tabular}{|c|c|c|c|c|c|}
\hline \multirow{2}{*}{ Age( in years) } & \multicolumn{2}{|c|}{ Elevated } & \multicolumn{2}{c|}{ Non elevated } & Total \\
\cline { 2 - 6 } & No & $\mathbf{\%}$ & No & $\mathbf{\%}$ & No \\
\hline Up to 20 & 1 & 3.6 & 19 & 26.4 & 20 \\
\hline $21-30$ & 5 & 17.9 & 17 & 23.6 & 22 \\
\hline $31-40$ & 6 & 21.4 & 16 & 22.2 & 22 \\
\hline $41-50$ & 8 & 28.6 & 15 & 20.8 & 23 \\
\hline $51-60$ & 4 & 14.3 & 4 & 5.6 & 8 \\
\hline$>60 \quad$ Total & 4 & 14.3 & 1 & 1.4 & 5 \\
\hline & 28 & 100.0 & 72 & 100.0 & 100 \\
\hline
\end{tabular}

TABLE - 2 ASSOCIATION OF RISK FACTORS WITH DRUG INDUCED LIVER DAMAGE

\begin{tabular}{|l|c|c|c|c|c|c|}
\hline \multirow{2}{*}{ Risk factor } & \multicolumn{2}{|c|}{$\begin{array}{c}\text { Elevated } \\
\text { (n=28) }\end{array}$} & \multicolumn{2}{c|}{$\begin{array}{c}\text { Non elevated } \\
\text { (n=72) }\end{array}$} & $\begin{array}{c}\text { P value } \\
\text { No }\end{array}$ & OR \\
& No & \% & No & \% & & \\
\cline { 2 - 5 } Male & 22 & 78.6 & 45 & 62.5 & 0.125 & 2.20 \\
\hline Female & 6 & 21.4 & 27 & 37.5 & 0.125 & 0.45 \\
\hline BMI <18 & 14 & 50.0 & 20 & 27.8 & 0.049 & 2.42 \\
\hline Alcohol & 13 & 46.4 & 11 & 15.3 & 0.001 & 4.81 \\
\hline $\begin{array}{l}\text { Chest X-ray } \\
\text { (severe) }\end{array}$ & 15 & 53.6 & 15 & 20.8 & 0.001 & 4.38 \\
\hline Serum Albumin & 15 & 78.6 & 15 & 20.8 & 0.000 & 13.93 \\
\hline
\end{tabular}

TABLE - 3 EFFECT OF ANTI-TB DRUGS ON LIVER $(n=100)$

\begin{tabular}{|l|c|l|l|l|}
\hline \multicolumn{1}{|c|}{ Study period } & \multicolumn{1}{|c|}{$\begin{array}{c}\text { Serum } \\
\text { bilirubin } \\
\text { (Mean } \pm \text { SD) }\end{array}$} & $\begin{array}{c}\text { AST } \\
\text { (Mean } \pm \text { SD) }\end{array}$ & $\begin{array}{c}\text { ALT } \\
\text { (Mean } \pm \text { SD) }\end{array}$ & $\begin{array}{c}\text { ALK } \\
\text { (Mean } \pm \text { SD) }\end{array}$ \\
\hline Baseline & $0.79 \pm 0.17$ & $23.43 \pm 6.84$ & $21.88 \pm 5.19$ & $64.75 \pm 20.04$ \\
\hline Week 2 & $0.87 \pm 0.22$ & $33.76 \pm 19.96$ & $34.24 \pm 26.11$ & $73.86 \pm 32.72$ \\
\hline Week 4 & $0.94 \pm 0.49$ & $50.34 \pm 65.45$ & $59.60 \pm 93.46$ & $82.75 \pm 38.16$ \\
\hline Week 8 & $0.88 \pm 0.29$ & $37.68 \pm 20.97$ & $36.74 \pm 24.54$ & $76.53 \pm 24.44$ \\
\hline $\begin{array}{l}\text { Significance } \\
\text { (Friedman test) }\end{array}$ & 0.176 & $\mathrm{P}<0.001$ & $\mathrm{P}<0.001$ & $\mathrm{P}<0.001$ \\
\hline
\end{tabular}



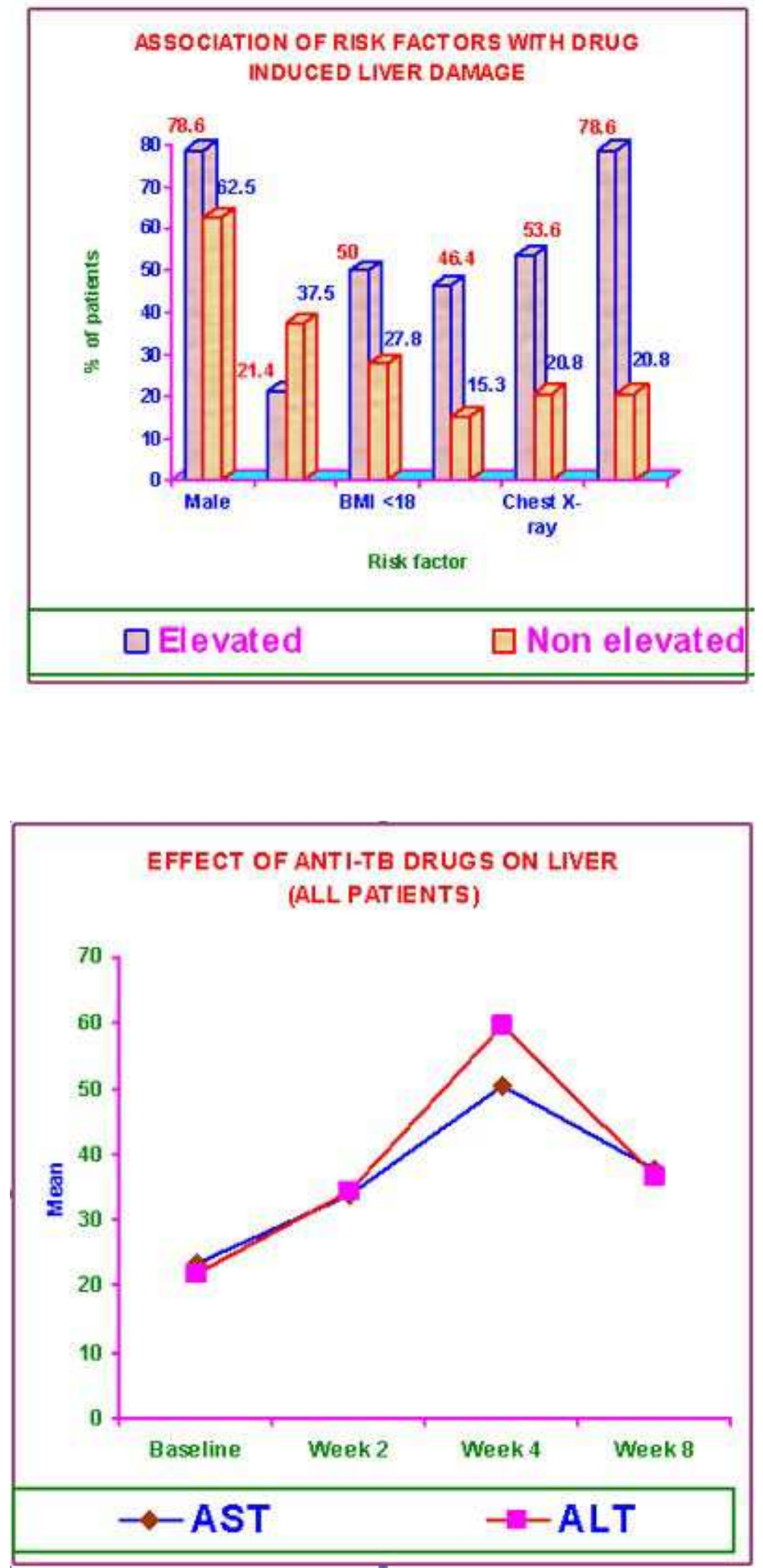


\section{ORIGINAL ARTICLE}

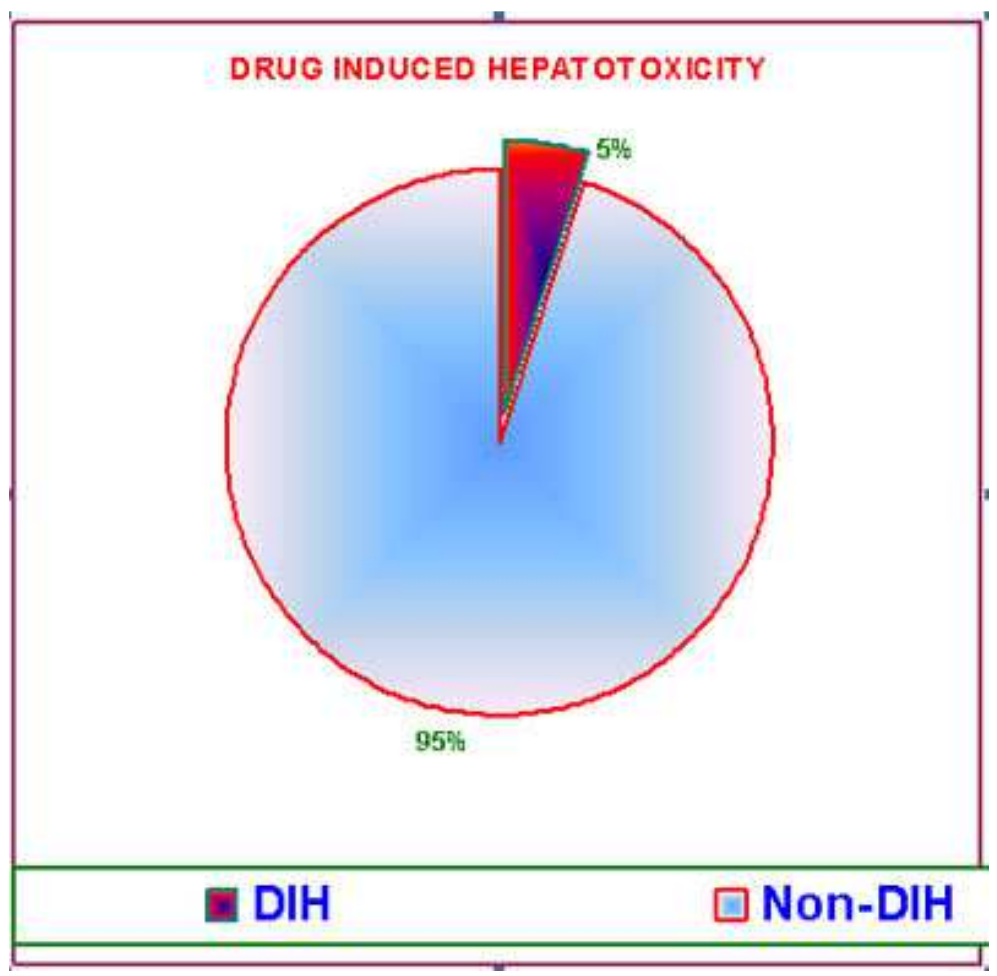

